\title{
Aktualisierte Empfehlungen zur Behandlung der Immunthrombozytopenie
}

\author{
Paul Imbach \\ Pädiatrische Onkologie-Hämatologie und Transplantation, Universitätskinderspital beider Basel, Basel, Schweiz
}

Viele unterschiedliche Faktoren der Immunthrombozytopenie bei Kindern und Erwachsenen erfordern Empfehlungen und Richtlinien der Diagnostik und Behandlung von Fachexperten.

Seit Hippokrates gibt es Berichte zu diesem Krankheitsbild, das als «Purpura» bezeichnet wurde. Ohne Kenntnis der Blutkomponenten war es nicht möglich, Blutungen zu differenzieren. Werlhof beschrieb 1751 das Syndrom «Blutung und Purpura», und erst nach der Entdeckung der Thrombozyten zwischen 1865 und 1883 durch Donné, Schultze und Bizzozero wurde von idiopathischer thrombozytopenischer Purpura (ITP) gesprochen.

1916 wurde die erste Splenektomie bei ITP durchgeführt - in Analogie zur hämolytischen Anämie -, wobei die Milz als Ursache der hohen Thrombozytenzerstörung erkannt wurde. Seither blieb die Splenektomie die erfolgreichste Behandlung bei ITP, obwohl der Verlust der Milz zeitlebens eine Immundefizienz hinterlässt.

Harrington's klassisches Experiment 1950/1951 [1] der Transfusion von Plasma von Patienten mit ITP bei gesunden Probanden - zuerst bei sich selbst! - bewies, dass ein humoraler Faktor die Thrombozytopenie bei den Probanden verursachte, und erst die Bestimmung von spezifischen Antikörpern durch Shulman 1965 zeigte die immunologische Ursache der Thrombozytenzerstörungen auf.

Fast gleichzeitig mit Harrington begann Wintrobe et al. 1951 [2] die erfolgreiche Behandlung der ITP mittels Verabreichung von Kortikosteroiden und adrenokortikotropem Hormon (ACTH). Seither ist Prednisolon eine Standardtherapie bei ITP, trotz der erheblichen Nebenwirkungen.

1981 folgte die erste Publikation zur Beobachtung, dass die Verabreichung von humanem Antikörperkonzentrat von Blutspendern bei Kindern mit akuter oder chronischer ITP - letztere Form mit oder ohne Splenektomie - zu einem unmittelbaren Thrombozytenanstieg führte [3]. In kontrollierten Studien erwies sich die Nebenwirkungsrate als gering gegenüber der Kortikosteroidbehandlung. Die immunologische Genese der ITP war jetzt auch im therapeutischen Bereiche bewiesen. Das Akronym «idiopathisch» wandelte sich $\mathrm{zu}$ «Immun»Thrombozytopenie, und da viele Patienten mit ITP keine Purpura aufweisen, wurde letztere Bezeichnung wegelassen.

Da die Immunpathogenese bei anderen autoimmunen Erkrankungen ähnlich ist, wurde die ITP zum Modell der polyklonalen und später auch der monoklonalen Antikörpertherapien. Weltweit wurden klinische Studien initiiert und Indikationen sowie Laborstudien der komplexen Wirkungsmechanismen der Antikörpertherapie von autoimmunen Erkrankungen durchgeführt [4].

2002 berichteten Stasi et al. [5] vom erfolgreichen Einsatz des monoklonalen Anti-CD20-Antikörpers bei Patienten mit chronischer ITP. Die Mehrzahl der Patienten hatte nach der Behandlung eine Monate dauernde Depletion von B-Zellen. Oft wurde die ITP nach einer längeren Periode mit hohen Thrombozytenwerten wieder manifest.

Parallel zur Anti-CD20-Option wurde die Wirksamkeit der anderen gezielten Behandlung der ITP - zunächst mit rekombinantem Thrombopoetin, dann mit Thrombopoetinrezeptor-Agonisten (TRA), die die Thrombozytenproduktion der Megakaryozyten steigern - in extensiven klinischen Studien bei Erwachsenen und Kinder mit chronischer ITP(mit oder ohne Splenektomie) untersucht. Auch haben McMillan et al. [6] experimentell gezeigt, dass in vitro Megakaryozytenkulturen von Patienten mit ITP eine unterschiedlich starke Hemmung der Thrombozytenproduktion auf wiesen. Heute ist die TRA-Therapie charakterisiert durch: Thrombozytenanstieg innerhalb 3-8 Tagen; Langzeitwirkung, solange die Therapie fortgesetzt wird; geringe Nebenwirkungsraten; bei Absetzen meist Wiederauftreten der ITP. Der hohen Kosten wegen können Antikörper- und TRA-Therapien nur eingeschränkt eingesetzt werden.

Damit nicht genug von verschiedenen neueren Aspekten: Die ersten Registry-Studien ab 1997 mit prospektiver Langzeitbeobachtung von mehreren Tausend Patienten von über 90 kooperierenden Zentren weltweit ( $w w w . i t p b a s e l . c h)$ führten unter anderem zur Änderung der Terminologie des Verlaufs der ITP: Heute sprechen

\section{KARGER}

() 2018 S. Karger GmbH, Freiburg

Fax +497614520714 
wir von neu diagnostizierter, von persistierender und von chronischer ITP. Die persistierende ITP erfordert den Ausschluss der sekundären Formen der ITP. Eine weitere Folge der Registry-Studien war, dass die Art von Blutungen, und nicht in erster Linie die niedrigeren Thrombozytenwerte, für die Therapie-Indikation wichtiger ist. Rodeghiero et al. [7] und später Neunert et al. [8] haben diese Ergebnisse publiziert.

Ein weiterer Faktor ist die Lebensqualität der Patienten. Seit 1984 werden systematische Untersuchungen bei Kinder und Erwachsenen - teilweise durch Selbsthilfegruppen der Erwachsenen - durchgeführt. Neben den unterschiedlichen Behandlungen mit Nebenwirkungen beeinflussen besonders Einschränkungen der Aktivitäten, das Fatigue-Syndrom, soziale Integration und andere mehr die Lebensqualität. Die Ungewissheit, welcher Patient eine Spontanheilung oder einen Langzeitverlauf der ITP entwickelt, bleibt bis heute bestehen. Auch ist die Korrelation zwischen Blutungssymptomen und Thrombozytenwerten individuell sehr unterschiedlich. Bei Patienten ohne schwere Blutungen zu Beginn oder im Verlauf der ITP kann «Wait and Watch» die bessere Alternative sein, sofern der verantwortliche Arzt im Gespräch mit Patient/Eltern diese Präferenz bevorzugt. Sonst ist eine aktive Behandlung, besonders bei Risiken oder Präsenz von schweren Blutungen, indiziert.
Ungelöst und damit Gegenstand für zukünftige Untersuchungen bleibt die zentrale Frage der Beeinflussung der Immunpathologie der ITP. Antikörpertherapie und Thrombozytenstimulation ändern/verbessern die komplexe Immundysfunktion der ITP, was sich ansatzweise in etwas höheren Raten von Spontanheilungen äußert. Aber: Wie erklärt sich Spontanheilung? Sind kombinierte Therapien oder eine monoklonale Antikörpertherapie zu Beginn der ITP zukünftige Optionen?

All diese oben aufgezeigten Faktoren und weitere mehr befeuerten seit 1991 die großen Anstrengungen von Expertengruppen, um Richtlinien und Empfehlungen zur Diagnostik und Behandlung/ Betreuung des individuellen Patienten zu erarbeiten und neue Erkenntnisse zu integrieren. Die vorliegende, den Verhältnissen 2018 angepassten deutsch-österreichisch-schweizerischen Empfehlungen unter Leitung von Axel Matzdorff sind ein willkommenes und wertvolles Beispiel.

\section{Disclosure Statement}

Kein Interessen-Konflikt.

\section{Literatur}

1 Harrington WJ, Minnich V, Holingsworth JW, Moore CV: Demonstration of a thrombocytopenic factor in the blood of patients with thrombocytopenic purpura. J Lab Clin Med 1951;38:1-10.

2 Wintrobe MM, Cartwright GE, Palmer JG, Kuhns WJ Samuesl LT: Effect of corticotrophin and cortisone on the blood in various disorders in man. AMA Arch Intern Med 1951;88:310-336.

3 Imbach P, Barandun S, d'Apuzzo V, Baumgartner C, Hirt A, Morell A, Rossi E, Schöni M, Vest M, Wagner HP: High-dose intravenous gammaglobulin for idiopathic thrombocytopenic purpura in childhood. Lancet 1981;i:1228-1231.
4 Imbach P (ed): Antibody Therapy: Substitution-Immunomodulation. Monoclonal Immunotherapy. Heidelberg, Springer, 2018, pp 1-342 (in press).

5 Stasi R, Stipa E, Forte V, Meo P, Amadori S: Variable patterns of response to rituximab treatment in adults with chronic idiopathic thrombocytopenic purpura. Blood 2002;99:3872-3873.

6 McMillan R, Wang L, Tomer A, Nichol J, Pistillo J: Suppression of in vitro megakaryocyte production by antiplatelet autoantibodies from adult patients with chronic ITP. Blood 2004;103:1364-1369.
7 Rodeghiero F, Stasi R, Gernsheimer T, Michel M, Provan D, Arnold DM, Bussel JB, Cines DB, Chong BH, Cooper N, Godeau B, Lechner K, Mazzucconi MG, McMillan R, Sanz MA, Imbach P, Blanchette V, Kühne T, Ruggeri M, George JN: Standardization of terminology, definitions and outcome criteria in immune thrombocytopenic purpura (ITP) of adults and children: Report from an International Working Group. Blood 2009; 113:2386-2393.

8 Neunert C, Lim W, Crowther M, Cohen A, Solberg L Jr, Crowther MA; American Society of Hematology: The American Society of Hematology 2011 evidencebased practice guideline for immune thrombocytopenia. Blood 2011;117:4190-4207. 